Trauma Berufskrankh 2016 $18: 116-124$

DOI 10.1007/s10039-016-0164-3

Online publiziert: 24. Mai 2016

(c) The Author(s) 2016 . This article is available at SpringerLink with Open Access

CrossMark

\author{
P. Gutsfeld' $\cdot$ S. Simmel ${ }^{2} \cdot$ E. Benning ${ }^{2} \cdot$ A. Brand ${ }^{3,4} \cdot$ P. Augat ${ }^{3,4}$ \\ ${ }^{1}$ Abteilung Unfallchirurgie, Klinikum Garmisch-Partenkirchen, Garmisch-Partenkirchen, Deutschland \\ ${ }^{2}$ Abteilung BG-Rehabilitation, Berufsgenossenschaftliche Unfallklinik Murnau, Murnau, Deutschland \\ ${ }^{3}$ Institut für Biomechanik, Berufsgenossenschaftliche Unfallklinik Murnau, Murnau am Staffelsee, \\ Deutschland \\ ${ }^{4}$ Institut für Biomechanik, Paracelsus Medizinische Privatuniversität Salzburg, Salzburg, Österreich
}

\title{
Orthesen in der Unfallchirurgie
}

Ziel dieses Beitrages ist es, die aktuelle Situation der Verwendung von Bandagen, Orthesen und Schienen in der Unfallchirurgie v. a. in der peri- und postoperativen Phase $\mathrm{zu}$ beleuchten. Aufschlussreich sind die Zahlen einer repräsentativen Umfrage des Instituts für Demoskopie Allensbach im Auftrag von Eurocom e. V. Danach tragen etwa $10 \%$ der deutschen Bevölkerung über dem 16. Lebensjahr zumindest zeitweise Bandagen oder Orthesen [1]. Fast ein Viertel davon bekam die Orthese nach einer Operation verschrieben. $\mathrm{Zu}$ den wichtigsten Erkenntnissen gehört, dass die Patienten die gesundheitliche Wirkung ihres Hilfsmittels in den meisten Fällen positiv bis sehr positiv einschätzen. Sie empfinden weniger Schmerzen und beobachten weniger Symptome, was sich entsprechend in einem geringeren Gebrauch von Schmerzmitteln äußert.

\section{Definition Orthese}

Eine Orthese ist eine äußerlich am Körper angebrachte Vorrichtung mit dem Zweck, strukturelle oder funktionelle Eigenschaften des Bewegungsapparates zu ändern. Die Orthese besteht aus einem oder mehreren Bauteilen und kann an oberen und unteren Gliedmaßen, Kopf, Hals oder Rumpf angewendet werden, um die neuromuskulären und skelettalen Systeme zu beeinflussen (ISO 8549-1).

Orthesen sind medizinische Hilfsmittel, die auf ärztliche Verordnung hin durch einen Orthopädietechniker oder industriell hergestellt werden. Hilfsmittel sind sachliche medizinische Leistungen - im
Gegensatz zu Heilmitteln, bei denen es sich um persönlich erbrachte medizinische Leistungen handelt, wie z. B. Physiotherapie, Sprachtherapie. Hilfsmittel haben Vorrang in der Verordnung $(\$ 9$ Wirtschaftlichkeit der Heilmittel-Richtlinie). Vor jeder Verordnung von Heilmitteln muss der behandelnde Arzt prüfen, ob entsprechend dem Gebot der Wirtschaftlichkeit das angestrebte Behandlungsziel auch durch eigenverantwortliche Maßnahmen des Patienten, durch eine Hilfsmittelversorgung oder durch Verordnung eines Arzneimittels qualitativ gleichwertig und kostengünstiger erreicht werden kann.

Orthesen werden in der Orthopädie und Unfallchirurgie meist zur temporären Behandlung muskuloskeletaler Erkrankungen oder Fehlfunktionen verwendet. Dabei beruht die Wirkung der Orthese im Wesentlichen auf ihrer mechanischen Funktion: Mobilisierung, Korrektur (von Stellung oder Bewegung), Führung, Stabilisierung, Entlastung oder Ruhigstellung. Die begriffliche Unterscheidung zwischen Bandagen, Orthesen und Schienen ist nicht immer einfach, da es zwischen diesen Gruppen fließende Übergänge gibt und eine allgemein anerkannte Nomenklatur bisher fehlt (- Tab. 1).

Bandagen bestehen in den meisten Fällen aus einer Kombination von Textilien und elastischen Einsätzen (Pelotten) und sind anatomisch vorgeformt. Die Wirkprinzipien von Bandagen sind: - Stabilisierung des Gelenkes insbesondere durch eine gezielte Anregung der
Muskelrezeptoren (Propriozeption, Sensomotorik),

- dosierte Kompression,

- intermittierende Massage und Wärme.

Orthesen sind dazu konzipiert definierte Haltungen herzustellen und zu sichern, Teile des Bewegungsapparates zu entlasten und Gelenkbewegungen zu führen, $\mathrm{zu}$ begrenzen oder zu verhindern. Aus der mechanischen Funktion folgt, dass Orthesen in wesentlichen Teilen aus festen, rigiden Materialien, z. B. Metallen und Kunststoffen, gefertigt werden. Mit Orthesen kann eine betroffene Körperregion daher vollständig ruhiggestellt, aber auch kontrolliert mobilisiert werden. Orthesen können immobilisieren, stabilisieren, mobilisieren, entlasten, redressieren (quengeln), korrigieren und ausgefallene Körperfunktionen ersetzen.

Schienen sind schließlich eine Sonderform von Orthesen, die der reinen Immobilisation dienen.

\section{Sprunggelenkorthesen}

\section{Sprunggelenkorthesen zur Immobilisierung}

Besteht die Indikation zur Immobilisation von Frakturen und Bandrupturen, insbesondere in der perioperativen Phase, kann eine solche vorübergehende Aufhebung der normalen Bewegungsumfänge entweder mit Gips (Gipsschalen, Gipsverbänden), aber auch mithilfe von immobilisierenden Orthesen erreicht werden. Orthesen zur Immobilisierung un- 


\begin{tabular}{|llll}
\hline Tab. 1 & Hauptgruppen orthopädischer Hilfsmittel mit Funktionsweise und Behandlungsziel \\
\hline & Bandagen & Orthesen & Schienen \\
\hline Funktionsweise & $\begin{array}{l}\text { Keine wesentliche } \\
\text { Begrenzung der Be- } \\
\text { wegungsumfänge } \\
\text { Verbesserung der }\end{array}$ & $\begin{array}{l}\text { Selektive Begrenzung } \\
\text { von Gelenkbewegun- }\end{array}$ & $\begin{array}{l}\text { Immobilisation des } \\
\text { Gelenkes }\end{array}$ \\
& $\begin{array}{l}\text { Sicherung definierter } \\
\text { Propriozeption und }\end{array}$ & Bewegungen & \\
& Sensomotorik & & \\
& Dosierte Kompression & & \\
\hline Behandlungsziel & Mobilisation & Frühe Mobilisation des & Protektion und Entlas- \\
& Beschleunigung des & Patienten & tung von Strukturen \\
& Heilungsverlaufs & Vermeidung von Immo- & Herstellen und Sichern \\
& Schmerzreduktion & bilisationsschäden & einer gewünschten \\
& & Entlastung und Schutz & Position des Gelenkes \\
& & von Strukturen & Schmerzreduktion \\
& & Schmerzreduktion & \\
\hline
\end{tabular}
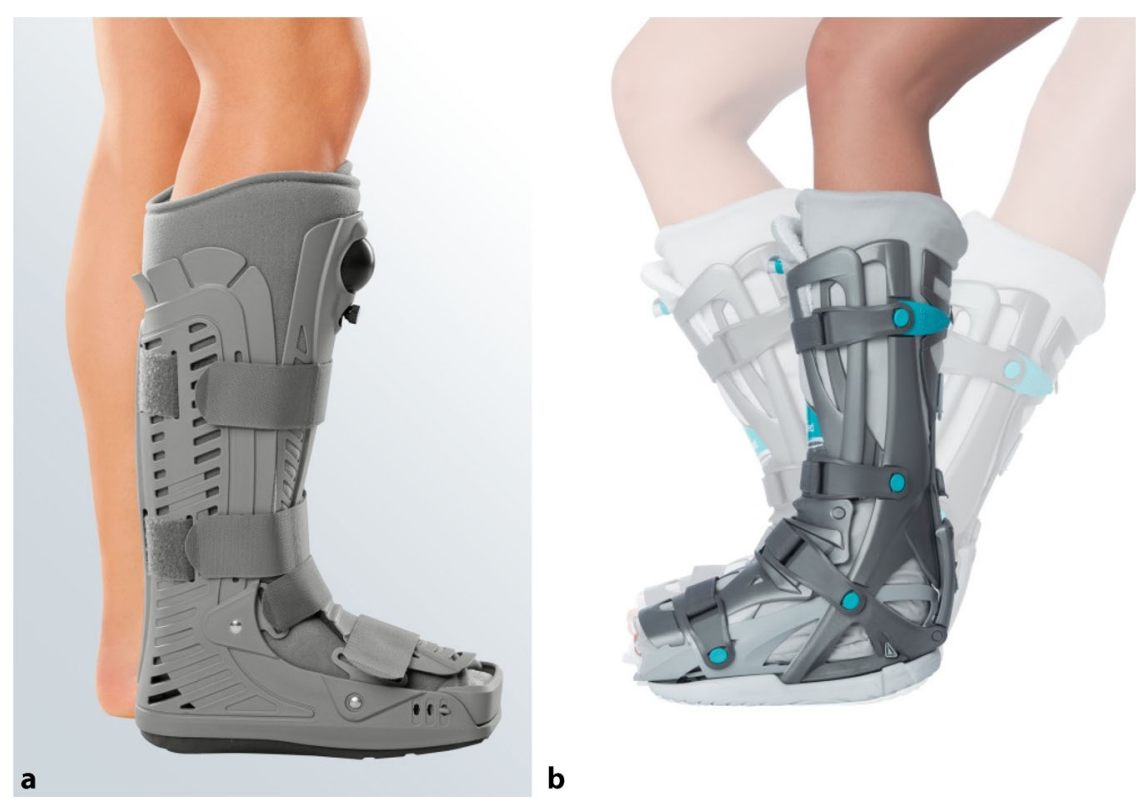

Abb. $1 \Delta$ a Sprunggelenkorthesen Protect CATwalker, MEDI (mit freundl. Genehmigung MEDI; www. medi.de). b Unterschenkelschiene mit limitierter Gelenkbeweglichkeit, VACOped (mit freundl. Genehmigung VACOhand OPED GmbH)

terbinden dabei wirkungsvoll Bewegungen in verschiedene Richtungen und stellen das Gelenk ruhig. Der Vorteil der Orthesen im Vergleich zu den Gipsruhigstellungen ist v. a. der Komfort für den Patienten, die Praktikabilität, die geringere Komplikationsrate und der Kostenaspekt, insbesondere unter dem Blickwinkel der Personalkosten.

Abzugrenzen sind Retentionen bei instabilen Situationen wie Luxationen des Sprunggelenkes, offene Frakturen oder auch Polytraumen. Hier sind Orthesen überfordert. Die sichere Retention einer Reposition erfordert entweder eine perfekte Gipsanwendung oder die Transfixation im Fixateur externe. und kostengünstiger Innenbezug erfüllt hygienische Anforderungen.

Die Lagerungsschiene kann durch eine streckseitige Halbschale ergänzt werden, sodass eine vollständige Unterschenkelorthese entsteht. Einfache Wundkontrolle und - falls möglich früheres Gehen unter Vollbelastung sind entscheidende Vorteile. Ferner kann in der funktionellen Unterschenkelorthese zur Nachbehandlung z. B. von Sprunggelenkfrakturen eine Beweglichkeit von -15 bis $+30^{\circ}$ freigegeben werden.

Die adäquate postoperative Nachbehandlung von Malleolarfrakturen ist ein häufiger Diskussionspunkt. Sie kann nicht nur das funktionelle Resultat beeinflussen, sondern auch die Zeit, die für eine adäquate Gehfähigkeit unter Verwendung von Gehstützen benötigt wird. Malleolarfrakturen, die funktionell ohne externe Stabilisierung behandelt wurden, zeigen im Vergleich zu einer Ruhigstellung im Gipsverband für 6 Wochen nach Osteosynthese keine signifikanten Unterschiede in den Langzeitresultaten [12].

Es hat sich jedoch erwiesen, dass durch eine frühzeitige Mobilisation in einer wechselbaren Orthese mit limitierter Bewegungsfreigabe die Compliance des Patienten verbessert und spätere Komplikationen der Gelenkruhigstellung, wie beispielsweise Thrombosen oder Knorpelschäden, verhindert werden können. Durch die Möglichkeit, auch in Spitzfußstellung zu fixieren, eignen sich immobilisierende Sprunggelenkorthesen in Verbindung mit einer Keilsohle auch zur Versorgung von Achillessehnenverletzungen.

Grundsätzlich darf die Immobilisation des Gelenkes nur nach genauer Indikationsstellung erfolgen. Eine Thromboseprophylaxe ist obligat. Besondere Polsterung, passgenaue Stabilität, eine Abrollsohle und die engmaschige klinische Kontrolle sind für Patienten mit Diabetes mellitus oder Polyneuropathien besonders wichtig. Notwendig ist eine ausführliche Aufklärung - am besten schriftlich - über mögliche Komplikationen, bei denen eine ärztliche Kontrolle unmittelbar erforderlich ist. Eine Hilfsmittelkontrolle im Folgetermin sollte das 
Produkt, den Sitz der Orthese und den Lokalbefund überprüfen.

\section{Definition Mobilisation}

Unter Mobilisation versteht man die kontrollierte Rückführung von verletzten, erkrankten bzw. unbeweglichen Gelenken in den physiologischen Bewegungsumfang.

\section{Sprunggelenkorthesen zur} Mobilisierung

Negative Auswirkungen einer Immobilisierung des Sprunggelenkes sind bekannt, sodass nach fibularen Bandrupturen eine konsequente Ruhigstellung nur initial einen Stellenwert hat. Nach Ausschluss einer Operationsindikation ist die konservative, frühfunktionelle Therapie angezeigt. In diesem Zusammenhang können funktionelle Orthesen einen wesentlichen Beitrag leisten. Unterschieden werden U-förmige Sprunggelenkorthesen (geringer Stabilisierungsgrad), Sprunggelenkorthesen mit funktionellem Zügel und Sohlenteil (mittlerer Stabilisierungsgrad) und konfektionierte Therapieschuhe als Unterschenkelorthese mit hohem Stabilisierungsgrad.

Bei posttraumatischen Veränderungen mit Schwellneigungen im Bereich des oberen und unteren Sprunggelenkes haben starre Orthesen in der Regel keine Indikation. Kompressionsbandagen mit eingearbeiteten Pelotten über der Gelenkkapsel und entlang des Sehnenverlaufs, auch im Bereich des Peritendineums der Achillessehne, können durchaus zur Verbesserung der Gangstabilität über propriozeptive Effekte und über den komprimierenden Effekt eine Besserung der lokalen Situation bewirken.

\section{Entlastende Unterschenkel- orthesen}

Ein besonderes Prinzip am Sprunggelenk sind die entlastenden Unterschenkelorthesen. Fersenentlastungsorthesen (nach Settner/Münch) entlasten gezielt den Kalkaneus und verlagern die axiale Belastung auf den Mittel- und Vorfuß

Trauma Berufskrankh 2016 · 18:116-124 DOI 10.1007/s10039-016-0164-3

(c) The Author(s) 2016

\section{P. Gutsfeld · S. Simmel · E. Benning · A. Brand · P. Augat}

\section{Orthesen in der Unfallchirurgie}

\section{Zusammenfassung}

Hintergrund. Die Verwendung von Orthesen als Hilfsmittel hat sich sowohl in der Unfallchirurgie als auch in der Akuttherapie etabliert. Es werden $22 \%$ der als Hilfsmittel verordneten Orthesen im Zusammenhang mit einer Operation verschrieben.

Fragestellung. Dargestellt werden die unterschiedlichen Anforderungen an und Ausgestaltungen von Orthesen insbesondere bei Sprunggelenkfrakturen, Verletzung des Kniegelenkes, handgelenknahen Unterarmfrakturen und instabilen Ellenbogenverletzungen. Ferner wird der Nutzen von valgisierenden Kniegelenkorthesen bei Varusgonarthrosen aufgezeigt. Entsprechend der unterschiedlichen Anforderung in der peri- und postoperativen Therapie werden die Orthesen in ihren Funktionsweisen dargestellt: Orthesen können immobilisieren, stabilisieren, mobilisieren, entlasten, redressieren (quengeln), korrigieren und ausgefallene Körperfunktionen ersetzen. Der Nutzen dieser Hilfsmittel ist darstellbar, die Studienlage aber insgesamt spärlich und auf spezielle Teilaspekte fokussiert.

Schlussfolgerungen. Ziel dieser Arbeit ist, das breite, in der Praxis genutzte Anwendungsspektrum dieser Hilfsmittel in der unfallchirurgischen Akuttherapie darzustellen und zu weiteren wissenschaftlich fundierten Arbeiten über den Nutzen der Verwendung von Orthesen in der Unfallchirurgie anzuregen.

\section{Schlüsselwörter}

Frühfunktionelle Therapie · Gonarthrose · Verletzung · Sprunggelenk · Ellenbogen

\section{Orthotic devices in trauma surgery}

\section{Abstract}

Background. The use of orthoses as assistive medical devices has become established in orthopedic surgery as well as in acute therapy. Of the medical orthotic devices prescribed, $22 \%$ are in connection with a surgical intervention.

Aim. This article provides an overview of requirements for the use of orthotic devices and the different configurations in particular for such applications as ankle fractures, injuries to the knee joint, fractures of the wrist and forearm and unstable elbow injuries. Furthermore, the benefits of knee braces for correction of varus osteoarthritis are presented. The modes of operation of the various orthotic devices are described according to the different requirements in perioperative and postoperative therapy.
Orthoses can be used to immobilize, stabilize, mobilize, relieve, reduce, correct and replace deficient body functions. Although the potential benefits of these assistive devices are often obvious, scientific evidence is sparse and typically focused on limited therapeutic aspects.

Conclusion. This article presents the wide range of applications of orthoses and similar medical aids in the daily practice of acute orthopedic surgery and is aimed at stimulating further research on the benefits of using orthotic devices in trauma surgery.

Keywords

Early functional therapy - Knee osteoarthritis . Injury · Ankle · Elbow
[4]. Entlastungsorthesen nach Allgöwer umfassen den mittleren und proximalen Unterschenkel und leiten die axiale Belastung über einen Gehbügel unter Entlastung des Sprunggelenkes und des Fußes weiter [24].

\section{Sprunggelenkorthesen zur Korrektur}

Peronäusschienen mit und ohne Gelenk ersetzen oder unterstützen die Extensoren im oberen Sprunggelenk. Klassische korrigierende Orthesen werden in der Kinderorthopädie bei komplexen Fußdeformitäten verwendet. 


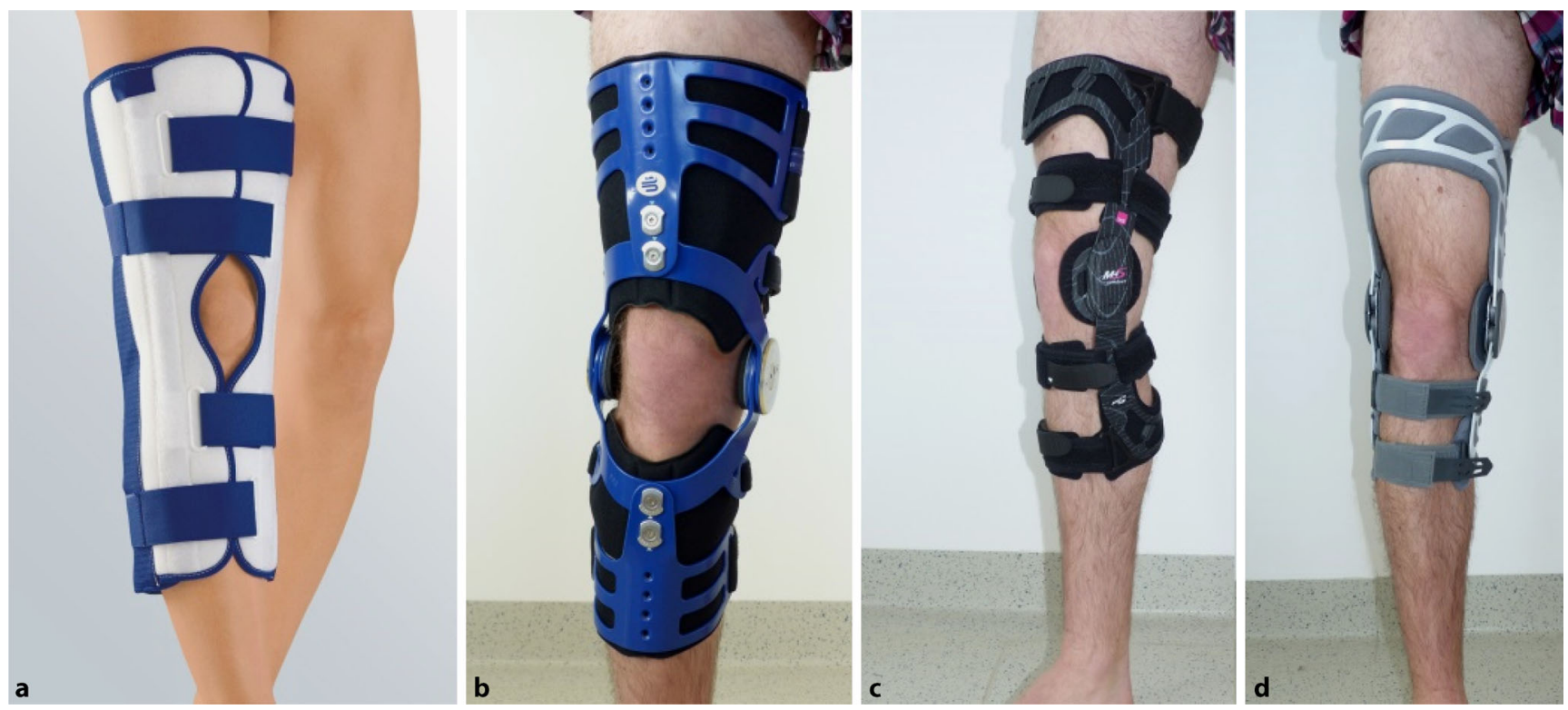

Abb. $2 \Delta$ Orthesen zur Stabilisierung des Kniegelenkes. a Immobilisierende Schiene (Medi Knieruhigstellungsschiene, mit freundl. Genehmigung MEDI; www.medi.de), b Stabilisierungsorthese nach Umstellungsosteotomie oder komplexen Bandinstabilitäten (MOS Genu, Bauerfeind), c Führungsorthese nach Kreuz- und Seitenbandruptur (M4.s Comfort, Medi), d Gonarthrosenorthese (4 Titude OA Nano, DJO)

\section{Kniegelenkorthesen}

Am Kniegelenk werden Orthesen wesentlich zur temporären Ruhigstellung, Stabilisierung und Entlastung eingesetzt. Dabei ergeben sich für Kniegelenkorthesen 3 verschiedene Anwendungskategorien [27]:

- prophylaktische,

- funktionelle und

- rehabilitative Orthesen.

Ziel der prophylaktischen Kniegelenkorthesen ist der Schutz vor Überlastungen bei Erhalt der physiologischen Gelenkfunktion. Funktionelle Knieorthesen haben die Aufgabe der Stabilisierung des Kniegelenkes und des Schutzes gefährdeter Strukturen, wie z. B. Kreuzbändern oder Meniskus. Rehabilitative Orthesen haben primär die Aufgabe, bei der Mobilisation des Kniegelenkes eine kontrollierte Bewegung zu ermöglichen und die verletzten Strukturen während der Rehabilitation vor mechanischer Überlastung zu schützen [27].

\section{Kniegelenkorthesen zur Immobilisierung}

Ist eine Protektion des Kniegelenkes notwendig, kann diese mittels Orthese erfolgen, sodass zwar das Knie, nicht aber der Patient in seiner Bewegung eingeschränkt wird. Die stabilste Form der Orthese am Kniegelenk ist das sog. „Fracture Brace“ das zur konservativen Behandlung stabiler Unter- oder Oberschenkelfrakturen nach dem Sarmiento-Prinzip eingesetzt werden kann [20]. Dabei wird die Fraktur stabilisiert, indem eine anpassbare Kunststoffschiene, meist bestehend aus 2 Halbschalen, Druck auf die Weichteile ausübt, die die Fraktur umgeben. Da die angrenzenden Gelenke dabei beweglich bleiben sollen, verfügt dieses Brace über eine einstellbare Beweglichkeit des Orthesengelenkes, die im Verlauf der Heilung mehr und mehr freigegeben werden kann. Die konservative Frakturbehandlung mit kniegelenküberbrückenden Braces erfordert eine klare Indikationsstellung und sorgfältige Überwachung des Heilungsverlaufs und der Patientencompliance, kann aber durchaus zu respektablen Heilungsergebnissen führen [21]. Für die Notfallversorgung und die perioperative Ruhigstellung erfolgt die Immobilisation in der konfektionierten Kniestreckschiene (• Abb. 2). Unterschiedliche Grundgrößen sowie einfache Klettverschlüsse machen sie individuell anpassbar. Neben der Si- cherung des Operationsergebnisses bis zur angeleiteten funktionellen Nachbehandlung stehen Schmerzreduktion und leichte Kompression im Vordergrund.

\section{Orthesen zur Mobilisierung}

In der frühfunktionellen Therapie haben die unterschiedlich ausgeformten Orthesen große Vorteile gegenüber der Gipsbehandlung oder den aufwendigen Funktionsgipsverbänden, da jene gezielt das verletzungsbedingte Defizit adressieren können. Die komplexe Biomechanik des Kniegelenkes mit seinem Roll-GleitMechanismus in Kombination mit der Schlussrotation sowie die unterschiedliche Kondylenbreite und -länge können von funktionellen Orthesen nur näherungsweise nachgebildet werden. Das momentane Drehzentrum des Kniegelenkes in Abhängigkeit von der Position verläuft auf einer Rastpolkurve und wird am besten durch den Kompromissdrehpunkt nach Nietert ([19]; • Abb. 3) wiedergegeben. Bei schlecht eingestellten Orthesen kann es zu hohen Fehlbelastungen des Kniegelenkes kommen. So kann eine geringe anteriore Verschiebung des Orthesengelenkes bereits Kräfte auf den Tibiakopf ausüben, die sich in einer Schubladenbewegung gegen das 


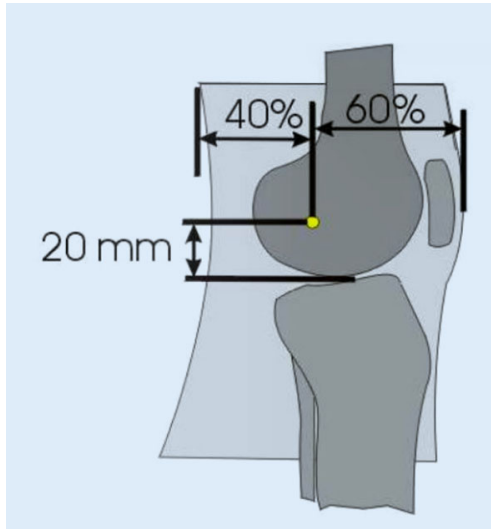

Abb. $3 \Delta$ Definition der Lage der Behelfsdrehachse im Kniegelenk

vordere Kreuzband auswirken. Aufgabe und Ziel in der technischen Orthopädie sind Knieführungsorthesen, die sich der Bewegung des Kniegelenkes möglichst genau anpassen.

In der Therapie der Seitenbandläsionen werden Knieorthesen verwendet, die durch Varus- oder Valguseinstellung und die Bewegungslimitierung das mediale bzw. laterale Kollateralband stabilisieren und entlasten. Diese Orthesen finden auch Einsatz in der postoperativen funktionellen Therapie von proximalen Tibiafrakturen. Ein weitverbreitetes Einsatzgebiet von Kniegelenkorthesen stellt die Nachbehandlung nach vorderer (VKB) und hinterer (HKB) Kreuzbandinsuffizienz dar. Die VKB-Insuffizienz ist durch die Komplexbewegung aus beginnender vorderer Translation und dann folgender Rotation gekennzeichnet. Vorderes oder hinteres Kreuzband sollen durch Orthesen nach dem 4-Punkt-Stabilisierungsprinzip entlastet werden, welche die proximale Tibia nach dorsal oder ventral redressieren. Durch die Vermeidung der Translationskomponente in der Orthese soll auch die Rotationsinstabilität reduziert werden. Nach Rekonstruktion des VKB kann bereits ab der ersten Woche eine Aufbelastung des Gelenkes unter Einschränkung des Bewegungsausmaßes $\left(0-0-90^{\circ}\right)$ erfolgen und die Limitierung der Gelenkbeweglichkeit ab der 4. bis 5. Woche freigegeben werden. Ab der 6 . bis 8 . Woche postoperativ kann auf die Orthese verzichtet werden.

Die Wirksamkeit der Orthesen in der Kreuzbandchirurgie ist nicht un- umstritten. Ein klinisch nachweisbarer Vorteil des Tragens einer Orthese bei der Rehabilitation nach VKB-Plastik lässt sich schwer nachweisen, und die Orthesennachbehandlung wird daher in der Literatur kritisch diskutiert [16]. Im Sinne des mechanischen Schutzes des Transplantates und dessen Fixierung sollte eine orthesenfreie Nachbehandlung immer eine sorgfältig abgestimmte Einzelfallentscheidung sein. Dabei müssen Schädigungsausmaß, Operationsverlauf, Transplantatauswahl und v. a. die Compliance und Erwartungshaltung des Patienten abgewogen werden [17]. Notwendig erscheint die Orthese bei recht häufig auftretenden Fällen von Begleitverletzungen der Kollateralbänder, der Menisken oder des Knochens.

Zur Frage der Sportprotektion und des Langzeittragens von Orthesen gerade im Sport lässt sich keine zusammenfasende Empfehlung finden. Während es Hinweise in Studien gibt, dass sich die Re-Rupturrate von VKB-Rekonstruktion durch das Tragen einer Orthese verringern lässt, bestehen doch erhebliche Zweifel gerade im Hinblick auf die fehlenden biomechanischen Eigenschaften der Orthese im Vergleich zur Komplexität der Bewegung des Kniegelenkes.

Bei der Ruptur des HKB besteht sowohl bei der konservativen Therapie als auch bei der operativen Nachbehandlung nach hinterer Kreuzbandplastik der Bedarf nach einer konsequenten posttraumatischen Ruhigstellung mit einer posterioren tibialen Support-Orthese (PTSSchiene) für einen Zeitraum von 6 Wochen. Danach erfolgt die Freigabe der Bewegung in einer funktionellen Orthese für die Behandlung von HKB-Insuffizienzen.

\section{Kniegelenkorthesen zur Gonarthrosebehandlung}

Als Ursachen für die Entstehung und das weitere Voranschreiten der Gonarthrose wird neben Übergewicht, Gelenktrauma und verringerter körperlicher Aktivität auch das veränderte anatomische Kniealignement als hoher Risikofaktor genannt [2]. Diese Varus- bzw. Valgusfehlstellung führt zu einer erhöhten mechanischen Belastung der Kniekompartimen- te, was letztendlich zu einer Degeneration des Gelenkknorpels führt. Aufgrund der natürlichen anatomischen Varusachse im Kniegelenk verlaufen etwa 60-70 \% der Gewichtsbelastung durch das mediale Kniekompartiment, was die erhöhte Prävalenz einer medial betonten Kniearthrose erklärt. Durch das daraus resultierende erhöhte Knieadduktionsmoment kommt es zu einer Verschmälerung des medialen Gelenkspalts und sekundär zu einem Knorpelverlust an der tibiofemoralen Kontaktfläche. Diese Erkenntnisse legen nahe, dass aus biomechanischer Betrachtungsweise die erhöhte Belastung der Kniekompartimente ein begünstigender Faktor für die Kniearthrose ist [10].

Eine Reduktion der Kniebelastung bzw. des Varusmoments bei medialer Gonarthrose mit konservativen Hilfsmitteln kann durch mechanisch wirkende Kniegelenkorthesen erreicht werden. Auf Basis des 3-Punkt-Entlastungsmechanismus soll durch seitlichen Druck auf das laterale Kniekompartiment eine funktionelle Achskorrektur der frontalen Kniegelenkachse erreicht werden. Andere Orthesen sollen eine Außenrotation des Beins bewirken, um dadurch eine erhöhte Stabilität im Kniegelenk zu erreichen [8]. Die Verwendung von Orthesen bei Patienten mit Gonarthrose führt zu einer biomechanisch nachweisbaren Entlastung des Kniegelenkes. Dies hat bei den Patienten eine mehr oder weniger stark ausgeprägte Reduktion des Schmerzempfindens und des damit verbundenen Schmerzmittelgebrauchs zur Folge [10].

\section{Kniegelenkorthesen zur Korrektur}

Das Zurückführen aus einer Fehlstellung wird als Korrektur bezeichnet. Demzufolge werden Orthesen zur Korrektur immer dann eingesetzt, wenn es darum geht, Fehlstellungen zu behandeln oder im postoperativen Verlauf zu vermeiden. Quengelorthesen erlauben ein stufenloses Korrigieren von Extensions- und Flexionsdefiziten am Knie. 

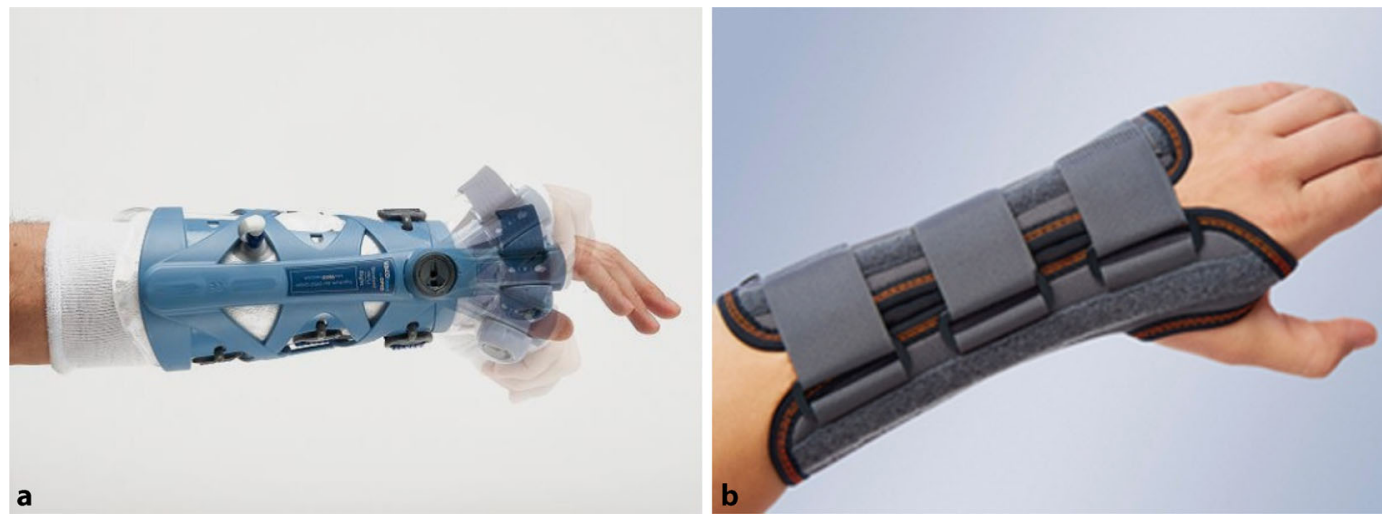

Abb. $4<$ a Handgelenkorthesen mit waschbarem Textilinlay und limitierter Beweglichkeit, VACOhand OPED GmbH (mit freundl. Genehmigung VACOhand OPED GmbH). b Konfektionierte Unterarmschiene, Manumed, MEDI (mit freundl. Genehmigung MEDI, www.medi.de)

\section{Kniegelenkbandagen}

Kniegelenkbandagen sind die mit am häufigsten abgegebenen Hilfsmittel und können bei posttraumatischen Schwellungszuständen und Ergüssen, aber auch bei peripatellaren Beschwerden bzw. Patellasubluxationen verwendet werden. Der Patient beschreibt oft einen besseren Halt im Kniegelenk, was auf eine Steigerung der Propriozeption über die komprimierende Hautstimulation zurückzuführen ist. Ein weiterer nachgewiesener Wirkeffekt ist die Kompression selbst. Die Orthesen werden jeweils als rein elastische Bandage, als Bandage mit Patellapelotte, meist ringförmig oder auch mit seitlichen führenden Scharniergelenken abgegeben. Bei allen Orthesen und Bandagen im Kniegelenkbereich ist in Anbetracht der Weichteilsituation die komprimierende Wirkung auf die Venen und damit die Gefahr einer Thrombose bei der Indikationsstellung mit einzubeziehen.

\section{Handgelenkorthesen}

Die Funktion von Handgelenkorthesen ist die Ruhigstellung nach Frakturen oder Weichteiltraumen sowie bei Arthrosen und Arthritiden. Weitere Funktionen der Handgelenkorthese sind die Verbesserung der Funktion durch Stabilisierung sowie die Prophylaxe bei Kontrakturen und Achsabweichungen. Handgelenkorthesen lassen typischerweise die Funktion der Grundgelenke der Finger frei (- Abb.4). Unterschieden werden folgende Vorrichtungen:

- Gipsverbände: Beim „echten Gips“ oder Weißgips handelt es sich um pulverisiertes Gipsmineral, das auf Mullbinden aufgetragen wird und bei der Verarbeitung mit Wasser unter Wärmeentwicklung zum festen Gips abbindet.

- Der Kunststoffgips oder Castverband besteht dagegen aus einem Netz von Kunstfasern, z. B. Glasfasern, das mit Kunstharz, z. B. Polyurethan, getränkt ist und unter Wassereinwirkung aushärtet. Vorteile gegenüber dem Weißgips sind schnelleres Aushärten, geringeres Gewicht und bessere Widerstandsfähigkeit. Nachteilig sind die deutlich höheren Kosten.

- Thermoplastische Schienen: Alternativ können vorgefertigte Schienen aus thermoplastischen Materialien mit niedriger Verarbeitungstemperatur angepasst werden.

- Funktionelle Schienen (Brace) aus elastischem Gewebe schützen vor extremen Bewegungen durch kräftige, formbare Verstärkungsschienen aus Aluminium. Die Innenseite besteht meist aus Baumwolle, und die Handhabung mittels Befestigungsschnallen oder Klettverschlüssen ist einfach. Zusätzlich finden Daumenverstärkungen zur Unterstützung und zum Halt des Daumens Verwendung. Diese Schienen lassen aber immer eine Restbeweglichkeit im Handgelenk zu, sodass die Ruhigstellung oder auch Retention nicht so sicher ist wie im Gips.

- Orthesen: Eine sehr stabile Ruhigstellung kann durch Orthesen mit Vakuumkissen erzielt werden. Zusätzlich besteht die Option der frühfunktionellen Behandlung durch ein einstellbares Gelenk in Höhe des Handgelenkes.

Im Hinblick auf die unfallchirurgische Akuttherapie stellt die Fraktur des distalen Radius das häufigste Anwendungsgebiet von Handgelenkorthesen dar. Die Literatur empfiehlt aktuell bei dislozierten Frakturen eine operative Versorgung mit Plattenosteosynthese. Die postoperative Behandlung, besonders der Effekt der Immobilisierung oder Mobilisierung, wird uneinheitlich gehandhabt und war bisher kaum Gegenstand wissenschaftlicher Studien [25]. Eindeutige Vorteile zeigt die Orthese bei der Patientenzufriedenheit im Hinblick auf Primärstabilität, Komfort und Hygiene.

Die Fraktur und deren Versorgung, insbesondere die kombinierten Bandläsionen, entscheiden über das notwendige Maß der Stabilität. In der Regel reicht es postoperativ nach bewegungsstabiler Osteosynthese aus, eine Schiene aus elastischem Gewebe und formbarer Verstärkung anzupassen. Dynamische Orthesen sind eine gute Alternative und sinnvolle Ergänzung zu herkömmlichen Hilfsmitteln.

\section{Ellenbogenorthesen}

Ellenbogenorthesen stabilisieren Ellenbogenflexion und -extension. Orthopädische Anwendungsschwerpunkte sind Lähmungen, posttraumatische Deformitäten, angeborene Fehlstellungen, Erkrankungen des rheumatischen Formenkreises und Überlastungssyndrome. In der Unfallchirurgie werden Orthesen v. a. bei instabilen Ellenbogenverletzungen und postoperativ eingesetzt. 

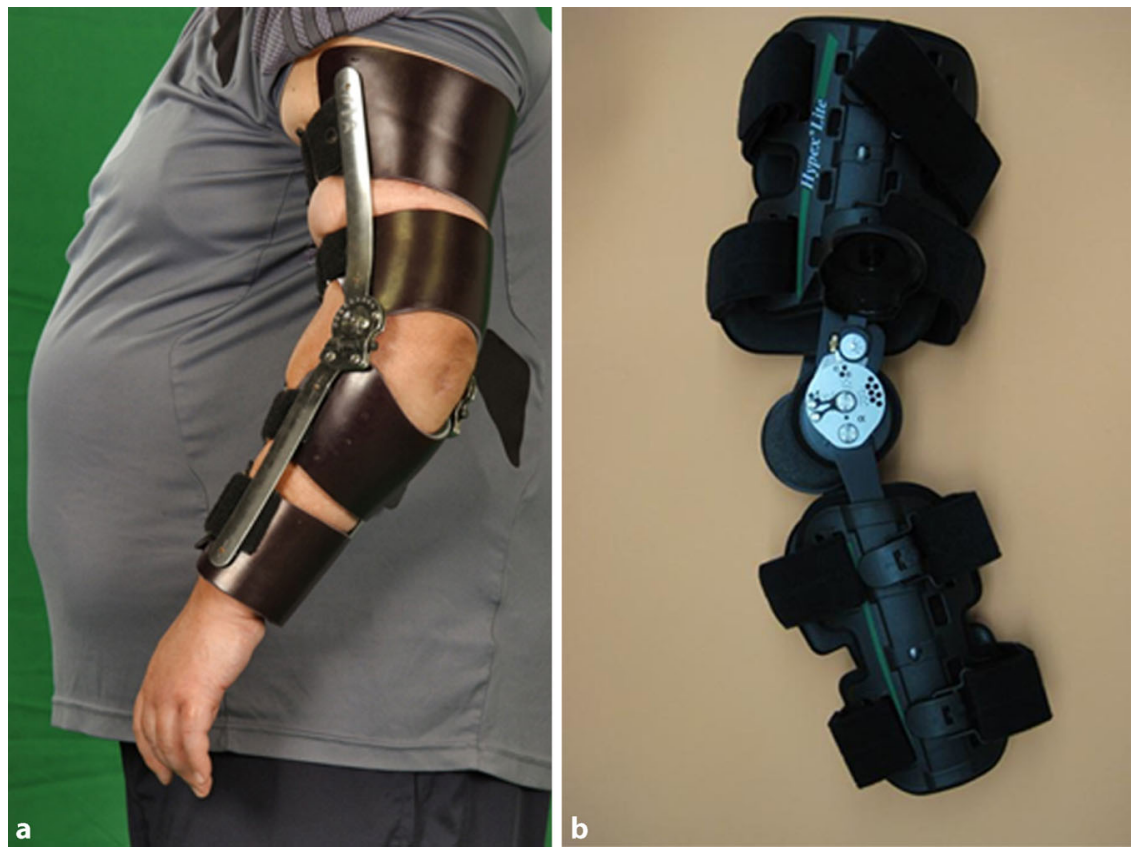

Abb. $5 \Delta$ Ellenbogenorthesen. a Individuell angefertigte statisch fixierbare Ellenbogenorthese.b Vorgefertigte statisch fixierbare Ellenbogenorthese, auch als Quengelschiene zu nutzen

\section{Statische Ellenbogenschiene}

Die statische Ruhigstellung des Ellenbogens mithilfe einer Lagerungsschiene ist nur als kurzfristige Maßnahme geeignet. Sie ist indiziert zur postoperativen $\mathrm{Ru}$ higstellung, zum Ausschalten schmerzhafter Bewegungen und zur Stabilisierung bei instabilem Ellenbogengelenk. Nach Reposition einer Ellenbogenluxation soll das Gelenk für maximal 1 Woche in $90^{\circ}$ Flexion ruhiggestellt werden, bevor der Wechsel auf eine Bewegungsorthese erfolgt. Abhängig von der Instabilitätsrichtung wird die Rotation des Unterarms fixiert: Sind die lateralen Stabilisatoren rupturiert, erfolgt die Ruhigstellung in Pronation, ist der mediale Komplex betroffen, in Supination. Sind beide Kollateralbänder betroffen, ist der Unterarm in Neutralstellung zu lagern [11,23].

\section{Dynamische Ellenbogenorthesen}

Bei den dynamischen Orthesen unterscheidet man zwischen Ellenbogenorthesen mit Flexions-/Extensionsbegrenzung, statisch fixierbaren Orthesen und Quengelschienen [26]. Ellenbogenorthesen mit Flexions-/Extensionsbegrenzung werden zur temporären Ruhigstellung und/oder zur funktionellen Nachbe- handlung bei akuten Kapsel-Band-Verletzungen am Ellenbogen eingesetzt, wenn innerhalb des funktionellen Bogens $\left(100^{\circ}\right.$-Regel nach Morrey) keine Reluxationstendenz besteht $[13,18$, 22]. Bewegungsorthesen sind auch zur postoperativen Behandlung indiziert, beispielsweise zum Schutz von Bandrekonstruktionen oder nach Ellenbogenarthroskopie [6, 7]. Sie unterstützen die Sicherheit des Patienten bei selbstständig durchgeführten Bewegungsübungen und reduzieren Varus- und Valgusscherkräfte [14]. Als Dauerversorgung dienen sie dem Schutz vor Unfällen bei Lähmungen (Plexusparese). Durch das einschränkbare Bewegungsausmaß kann dieser Orthesentyp individuell und gezielt je nach den geforderten Ansprüchen eingestellt werden. Grundsätzlich kann eine Ellenbogenorthese mit Flexions-/ Extensionsbegrenzung auch als statisch fixierbare Orthese oder als Lagerungsschiene genutzt werden.

\section{Quengelschienen und statisch fixierbare Orthese}

Quengelschienen und statisch fixierbare Orthesen können bei leichteren Ellenbogengelenkkontrakturen z. B. nach Verbrennungen, als Folge spastischer
Lähmungen oder intraartikulären Narbenbildungen, aber auch postoperativ nach Arthrolyse oder Narkosemobilisation eingesetzt werden $[9,15]$. Bei der Quengelschiene wird die Gelenkkontraktur durch Dehnung der Gelenkkapsel, der Bänder und der Muskulatur verringert bzw. aufgehoben. Dieses geschieht durch einen dauerhaften äußeren Zug in die Flexion oder Extension. Die Anwendung von Quengelschienen wird kontrovers diskutiert, da die kontinuierliche statische Kraft, die auf das Gelenk wirkt, zu einer inflammatorischen Reaktion des Gelenkes führen kann [5]. Die Quengeltherapie ist daher kontinuierlich zu überwachen. Quengelschienen sind bei entzündlichen Gelenkschwellungen kontraindiziert.

Die statisch fixierbare Orthese kann in einstellbaren Flexions- und Extensiongraden blockiert werden ( $\bullet$ Abb. 1, 2 und 5). Die Therapie zielt auf eine allmähliche Dehnung der Gelenkkapsel ab - im Gegensatz zur Dauerdehnung mittels Quengelschiene. Bei der statisch fixierbaren Orthese wirkt zu Beginn der Behandlung ebenfalls eine konstante statische Kraft, die allerdings nicht dauerhaft erhalten bleibt. Der Patient kontrolliert die Dauer und die Intensität der Dehnung selbst, wobei er angewiesen werden muss, selbstdiszipliniert einem strengen Nachbehandlungskonzept $\mathrm{zu}$ folgen. Morrey hat ein Nachbehandlungsschema entwickelt und beschrieben, das den biologischen Problemen, die eine kontinuierliche Dehnung inflammatorisch veränderten Gewebes verursacht, Rechnung trägt [5].

\section{Ellenbogenbandagen}

Ellenbogenbandagen sind bei chronischen postoperativen oder posttraumatischen Reizzuständen, bei Arthrose, Arthritis, Epikondylitis, Bursitis olecrani, rheumatoiden Erkrankungen oder lokalen Insertionstendinopathien indiziert. Durch die lokale Kompression kommt es sowohl zu einer Veränderung der Krafteinleitung der Muskulatur durch mechanische Kompression als auch zu einer Stimulation von Propriorezeptoren. Durch die eingearbeiteten Pelotten kann es zur Muskelstimulation und 
Muskelfazilitation kommen. Bei Epicondylitis humeri radialis (Tennisellenbogen) oder ulnaris (Golferellenbogen) können Epikondylitisspangen begleitend zur konservativen Therapie eingesetzt werden. Die Kompression durch die vorkonfektionierte Stabilorthese mit lokaler Druckpelotte auf dem entsprechenden Sehnenansatz am Ellenbogen kaudal des Epikondylus ist in der Druckstärke einstellbar. Sie vergrößert und verschiebt das Belastungszentrum und ändert die Zugrichtung der Sehne [3].

\section{Fazit für die Praxis}

- Orthesen sind in der unfallchirurgischen Akuttherapie sind ein geeignetes Hilfsmittel. Schmerzen werden reduziert, und Schmerzmittel können eingespart werden.

- Orthesen können immobilisieren, stabilisieren, mobilisieren, entlasten, redressieren (quengeln), korrigieren und ausgefallene Körperfunktionen ersetzen.

- In der frühfunktionellen Therapie haben die unterschiedlich ausgeformten Orthesen große Vorteile gegenüber der Gipsbehandlung oder den aufwendigen Funktionsgipsverbänden, da sie gezielt das verletzungsbedingte Defizit adressieren können. Eine Immobilisation darf nur nach genauer Indikationsstellung erfolgen.

- Eine ausführliche Aufklärung über mögliche Komplikationen ist erforderlich.

- Bei instabilen Situationen wie Luxationen des Sprunggelenkes oder Kniegelenkes, offenen Frakturen oder auch Polytraumen sind Orthesen im Einzelfall überfordert. Die sichere Retention einer Reposition erfordert entweder eine perfekte Gipsanwendung, besser die Transfixation im Fixateur externe.

\section{Korrespondenzadresse}

\section{Prof. Dr. P. Augat}

Institut für Biomechanik, Berufsgenossenschaftliche Unfallklinik Murnau

Prof.-Küntscher-Str. 8, 82418 Murnau am

Staffelsee, Deutschland

biomechanik@bgu-murnau.de

\section{Einhaltung ethischer Richtlinien}

Interessenkonflikt. P. Gutsfeld, S. Simmel, E. Benning, A. Brand und P. Augat geben an, dass kein Interessenkonflikt besteht.

Dieser Beitrag beinhaltet keine von den Autoren durchgeführten Studien an Menschen oder Tieren.

Open Access. This article is distributed under the terms of the Creative Commons Attribution 4.0 International License (http://creativecommons.org/ licenses/by/4.0/), which permits unrestricted use, dis tribution, and reproduction in any medium, provided you give appropriate credit to the original author(s) and the source, provide a link to the Creative Commons license, and indicate if changes were made.

\section{Literatur}

1. Anonymous (2014) Mehr Lebensqualität, weniger Schmerzen: Nutzung und Wirksamkeit orthopädischer Hilfsmittel. www.presseportal.de/pm/ 50795/2810570.Zugegriffen: 4. April 2016

2. Arden N, Nevitt MC (2006) Osteoarthritis: epidemiology. Best Pract Res Clin Rheumatol 20:3-25

3. Baumgartner R (2007) Epicondylitisspange. In: Greitemann B (Hrsg) Grundkurs der technischen Orthopädie. Thieme, Stuttgart

4. Baumgartner R, Greitemann B (2015) Grundkurs Technische Orthopädie. Thieme, Stuttgart

5. Beickert RBV (2003) Arthrolyse des Ellengelenks. Trauma Berufskrankh 5:41-54

6. Burkhart KHB, Wegmann K, Müller L (2012) Luxationen und Bandverletzungen am Ellenbogen und Unterarm. Orthop Unfallchir 7:435-462

7. Dehlinger Fl, Ries C, Hollinger B (2014) LUCL reconstruction using a triceps tendon graft to treat posterolateral rotatory instability of the elbow. Oper Orthop Traumatol 26:414-429

8. Dessery Y, Belzile EL, Turmel S et al (2014) Comparison of three knee braces in the treatment of medial knee osteoarthritis. Knee 21:1107-1114

9. Haselhuhn KD (2015) Einsteifung nach Ellenbogenverletzung. Trauma Berufskrankh 17:140-147

10. Henriksen M, Creaby MW, Lund $\mathrm{H}$ et al (2014) Is there a causal link between knee loading and knee osteoarthritis progression? A systematic review and meta-analysis of cohort studies and randomised trials. BMJ Open 4:e005368

11. Hollinger B, Dehlinger F, Franke S(2014) Diagnostik und Therapie der ligamentären Ellenbogeninstabilitäten. Obere Extremität:147-155

12. Honigmann P, Goldhahn S, Rosenkranz J et al (2007) Aftertreatment of malleolar fractures following ORIF-functional compared to protected functional in a vacuum-stabilized orthesis: a randomized controlled trial. Arch Orthop Trauma Surg 127:195-203
13. Kusche H, Gutsfeld P, Bühren V (2012) Bandverletzungen des Ellenbogengelenks. OP-Journal 28:48-56

14. Lenich A, Göpel U, Siebenlist S, Imhoff A (2014) Diagnostische und therapeutische Ellenbogenarthroskopie. Orthop Unfallchir 9:255-275

15. Lichtenberg S (2014) Ellenbogenkontraktur. Obere Extremität 9:163-171

16. Mayr HO, Stueken P, Munch EO et al (2014) Brace or no-brace after ACL graft? Four-year results of a prospective clinical trial. Knee Surg Sports Traumatol Arthrosc 22:1156-1162

17. Mcdevitt ER, Taylor DC, Miller MD et al (2004) Functional bracing after anterior cruciate ligament reconstruction: a prospective, randomized, multicenter study. Am J Sports Med 32:1887-1892

18. Morrey BF (2009) Functional evaluation of the elbow. the elbow and its disorders, 4. Aufl. Saunders, Philadelphia, S80-92

19. Nietert M (1977) The human knee-joint as a biomechanical problem (author's transl). Biomed Tech (Berl) 22:13-21

20. Sarmiento A, Gersten LM, Sobol PA et al (1989) Tibial shaft fractures treated with functional braces. Experience with 780 fractures. J Bone Joint Surg Br 71:602-609

21. Sarmiento A, Sharpe FE, Ebramzadeh E et al (1995) Factors influencing the outcome of closed tibial fractures treated with functional bracing. Clin Orthop Relat Res:8-24

22. Siebenlist S, Biberthaler P (2015) Akute KapselBand-Verletzungen des Ellenbogens. Trauma Berufskrankh 17:132-139

23. Siebenlist S, Lucke M, Lenich A, BiberthalerP(2015) Ellenbogenluxation - wann, was fixieren? Trauma Berufskrankh 17:32-38

24. Specht J, Scmitt M (2008) Technische Orthopädie. Springer, Heidelberg

25. Stuby FM, Dobele S, Schaffer SD et al (2015) Early functional postoperative therapy of distal radius fracture with a dynamic orthosis: results of a prospective randomized cross-over comparative study. PLoS ONE 10:e0117720

26. Winkler W (2006) Orthopädietechnische Versorgungsmöglichkeiten im Bereich des Ellenbogens. Man Med 44(4):295-297

27. Wolter M (2009) Die Nachbehandlung der vorderen Kreuzbandplastik mit und ohne Orthese - eine Vergleichsstudie. Dissertation, LudwigMaximilians-Universität, München 
Hier steht eine Anzeige.

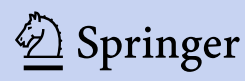

